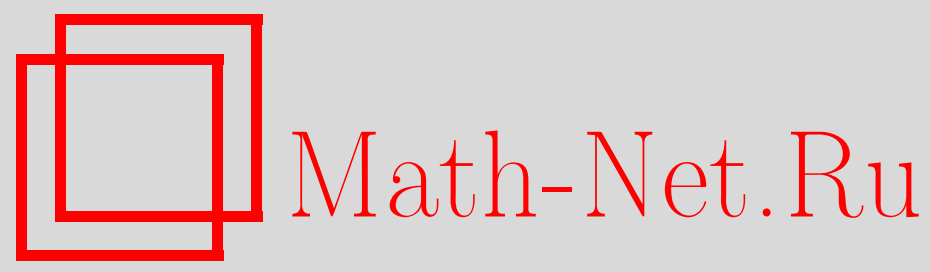

Д. Н. Азаров, О нильпотентной аппроксимируемости свободных произведений свободных групп с циклическим объединением, Матем. заметки, 1998, том 64, выпуск 1, 3-8

DOI: https://doi.org/10.4213/mzm1366

Использование Общероссийского математического портала Math-Net.Ru подразумевает, что вы прочитали и согласны с пользовательским соглашением http://www.mathnet.ru/rus/agreement

Параметры загрузки:

IP : 54.80 .97 .219

26 апреля 2023 г., $17: 49: 02$

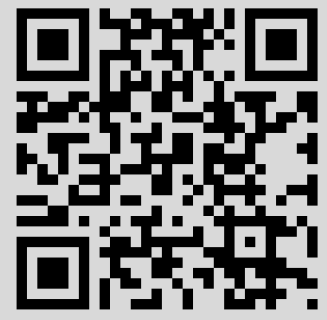




\section{О НИЛЬПОТЕНТНОЙ АППРОКСИМИРУЕМОСТИ СВОБОДНЫХ ПРОИЗВЕДЕНИЙ СВОБОДНЫХ ГРУПП С ЦИКЛИЧЕСКИМ ОБЪЕДИНЕНИЕМ}

\section{Д. Н. Азаров}

Пусть $G$ - свободное произведение свободных групп с циклическим объединением. В этой заметке доказьвается критерий аппроксимируемости группы $G$ конечными $p$-группами. Устанавливаются также другие аппроксимационные свойства группы $G$.

Библиограффия: 9 названий.

1. Введение. Пусть $A$ и $B$ - свободныегрупшы, $h$ и $k$-неединичные элементы групш $A$ и $B$ соответственно, $G=(A * B ; h=k)$ - свободное произведение групп $A$ и $B$ с объединенными циклическими подгруппами $H=(h)$ и $K=(k)$. Г. Баумслаг [1] доказал, что группа $G$ финитно аппроксимируема. В работе Дж. Дайер [2] доказьвается, что группа $G$ финитно апшроксимируема относительно сопряженности. Браннер, Бернс и Солитер [3] доказали, что все конечно порожденные подгруппы этой групшы финитно отделимы. В данной заметке рассматриваются другие аппоксимационные свойства группы $G$. В частности, устанавливается критерий аппроксимируемости группы $G$ конечньпи $p$-группами.

ТЕОрема 1. Пусть $A$ и $B$ - свободные группь, $h u k$-неединичные әлементы әрупп $A$ и $B$ соответственно и $G=(A * B ; h=k)$. Пусть $m$ u $n$ - наибольиие полохстельные числа такие, что уравнения $x^{m}=h$ и $y^{n}=k$ разрешимы в группах $A$ и В соответственно. Если одно из чисел $m$ или $n$ равно 1 , то для любого простого числа р группа $G$ аппроксимируется конечными р-группами. Если жсе $m>1$ u $n>1$, то группа $G$ аппроксимируется конечными р-группами тогда $u$ только тогда, когда числа $m$ и $п$ являются степенями числа $р$.

Заметим, что достаточное условие апшроксимируемости группы $G$ конечными $p$-группами было получено Гильденхьюзом [4]. Используя обозначения из теоремы 1 , результат Гильденхьюза можно сформулировать следующим образом: если числа $m$ и $n$ являются степенями простого числа $p$, то группа $G$ аппроксимируется конечными $p$-группами.

Пусть $\pi$ - множество простых чисел. Напомним, что конечная группа называется $\pi$-группой, если множество всех простых делителей ее порядка содержится в множестве $\pi$.

Следующая теорема дополняет отмеченньй выше результат Баумслага. 
Теорема 2. Пусть $A, B, h, k, m, n$ такие же, как и в теореме 1. Если множество простых чисел $\pi$ непусто и содержит все простые делители чисел $m$ и $n$, то группа $G$ аппроксимируется конечными разрешимыми т-группами.

Поскольку конечная $p$-группа является нильпотентной, из аппроксимируемости данной групшы конечными $p$-группами следует ее нильпотентная аппроксимируемость. Для рассматриваемой нами групшы $G$ имеет место и обратное утверждение.

Tеорема 3. Пусть $A, B, h, k$ такие жме, как и в теореме 1. Группа $G$ anпроксимируется нильпотентными группами тогда и только тогда, когда она аппроксимируется конечными р-группами для подходящего простого числа р.

Доказательства теорем 1, 2 и 3 приведены в п. 3. В п. 2 изложены некоторые предварительные утверждения.

2. Предварительные сведения. В работе Стиба [5, лемма 1] доказьвается, что для любого неединичного элемента $c$ свободной группы $F$ и для любогоположительного целого числа $l$ существует гомоморфизм группы $F$ на конечную группу, переводящий элемент $c$ в элемент порядка $l$. Почти дословное повторение доказательства этой леммы позволяет получить следующее более общее утверждение.

ПРЕДЛОЖЕНИЕ 1. Пусть группа $F$ аппроксимируется конечно порожденными нильпотентными группами без кручения, $c$ - неединичный әлемент әруппы $F$. Тогда для каждого положительного челого числа l существует гомоморфизм $\varphi$ группы $F$ на конечную нильпотентную группу $V$ такой, что $|c \varphi|=l$. Более того, если некоторое непустое мнохсество простых чисел $\pi$ содержит все простые делители числа $l$, то мохно считать, что группа $V$ является конечной нильпотентной $\pi$-группой.

Пусть $F$ - произвольная группа, $\pi$ - непустое множество простых чисел. Подгруппа $H$ групшы $F$ называется $\pi$-отделимой, если для любого элемента $x \in F \backslash H$ найдутся число $p \in \pi$ и гомоморфизм $\varphi$ групшы $F$ на конечную $p$-группу, отделяюший $x$ от $H$, т.е. такой, что $x \varphi \notin H \varphi$.

ПРЕДЛОЖЕНИЕ 2. Пусть $F-$ свободная группа, H-неединичная ииклическая подгруппа группы $F$ с порождающим әлементом $h, m$ - наибольшее положительное число такое, что уравнение $x^{m}=h$ разрешимо в группе $F$. Пусть $\pi-$ непустое мнохество простых чисел. Подгруппа $H$ является $\pi$-отделимой тогда и только тогда, когда множество $\pi$ содержит все простые делители числа т.

ДокАЗАтЕЛьСтво. Необходимо доказать только достаточность. Пусть множество простых чисел $\pi$ содержит все простые делители числа $m$. Покажем, что для произвольного элемента $x \in F \backslash H$ существует простое число $p \in \pi$ и гомоморфизм групшы $F$ на конечную $p$-группу, отделяющий $x$ от $H$.

Пусть $G_{F}(H)$ - централизатор подгруппы $H$ группы $F$. Возможны следующие случаи:

1) $x \notin G_{F}(H)$;

2) $x \in G_{F}(H) \backslash H$.

В случае 1$)$ коммутатор $[x, h]$ отличен от 1 . Зафиксируем число $p \in \pi$. Поскольку свободная группа $F$ аппроксимируется конечными $p$-группами, существует гомомор- 
физм групшы $F$ на конечную $p$-групшу, ядро которого не содержит $[x, h]$. Очевидно, что этот гомоморфизм отделяет $x$ от $H$.

В случае 2), очевидно, подгруппа $G_{F}(H)$ является циклической. Пусть $c$ - ее порождаюший элемент. Тогда $h=c^{m}, x=c^{k}$, где $k$ - целое число. Поскольку $x \notin H$, то $k$ не делится на $m$. Поэтому число $k$ не делится на некоторьй делитель числа $m$ вида $p^{s}$, где $p$ - простое число, $s$ - целое положительное число. По предложению 1 существует гомоморфизм $\varphi$ группы $F$ на конечную $p$-группу такой, что $|c \varphi|=p^{s}$. Так как $m$ делится на $p^{s}$ и $k$ не делится на $p^{s}$, то $h \varphi=1$ и $x \varphi \neq 1$. Таким образом, $x \varphi \notin H \varphi$, т.е. элемент $x$ отделяется от подгрупшы $H$ некоторьп гомоморфизмом группы $F$ на конечную $p$-группу. Причем, поскольку $p$ делит $m$ и множество $\pi$ содержит все простые делители числа $m$, то $p \in \pi$. Предложение доказано.

ПРЕДЛОЖЕНИЕ 3. Пусть $\pi$ - непустое множсество простых чисел, Я - класс

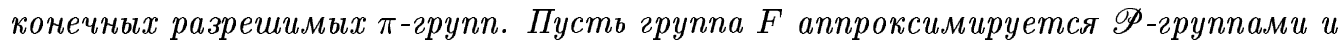

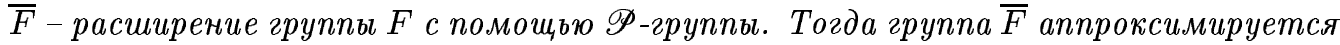
$\mathscr{P}$-группами.

ДокАЗАТЕЛьСтво. Легко проверить, что если $1 \leqslant K \leqslant H \leqslant G-$ произвольный субнормальньй ряд и $G / H, H / K \in \mathscr{P}$, то существует нормальная подгрупша $L$ группы $G$ такая, что $L \leqslant K$ и $G / L \in \mathscr{P}$. (В качестве подгрупшы $L$ можно взять пересечение всех подгрупп группы $G$, сопряженных с $K$.) По лемме 1.5 из [6] група $\bar{F}$ аппроксимируется $\mathscr{P}$-группами.

Пусть $A$ и $B$ - произвольные группы, $h$ и $k$ - элементы групп $A$ и $B$ соответственно, причем порядки этих элементов равны. Пусть, далее, $G=(A * B ; h=k)$ - свободное произведение этих групп с циклическим объединением. Для дальнейшего изложения нам потребуется следующая конструкция, восходящая к Баумслагу. Пусть $M$ и $N-$ нормальные подгрупш групп $A$ и $B$ соответственно, причем порядки элементов $h$ и $k$ по модулю этих подгрупп совпадают. Тогда можно рассмотреть свободное произведение с объединением $G_{M N}=(A / M * B / N ; h M=k N)$ и гомоморфизм $\rho_{M N}: G \rightarrow G_{M N}$, продолжающий естественные гомоморфизмы $A \rightarrow A / M$ и $B \rightarrow B / N$.

ПРЕДЛОЖЕНИЕ 4. Пусть группь $A$ и $B$ аппроксимируются конечно порожденными нильпотентными группами без кручения, $H=(h)$ и $K=(k)$ - бесконечные ииклические подгруппь групп $A$ и $B$ соответственно. Пусть, далее, $\pi$ - непустое множество простых чисел. Если подгруппы $H$ и $K$ т-отделимы в группах $A$ и $B$ соответственно, то группа $G=(A * B ; h=k)$ аппроксимируется конечными разрешимыми т-группами.

ДокаЗАтЕльство. Заметим, что сомножители $A$ и $B$ аппроксимируются конечными $p$-группами для любого простого числа $p$, поскольку эти групы по условию апшроксимируются конечно порожденньми нильпотентньпи группами без кручения.

Пусть $g$ - неединичный элемент группы $G$. Покажем, что существует гомоморфизм $\varphi$ группы $G$ на конечную разрешимую $\pi$-группу такой, что $g \varphi \neq 1$. Рассмотрим несократимую запись элемента $g$ :

$$
g=x_{1} x_{2} \cdots x_{r}
$$

Предположим сначала, что $r=1$. Тогда без потери общности можно считать, что $g \in A$. Зафиксируем число $p \in \pi$. Так как группа $A$ аппроксимируется конечными 
$p$-группами, существует нормальная подгруппа $M$ конечного $p$-индекса группы $A$, не содержашая элемент $g$. Поскольку группа $B$ аппроксимируется конечно порожденными нильпотентньпи группами без кручения и порядок элемента $h M$ групшы $A / M$ является степенью числа $p$, то по предложению 1 существует нормальная подгруппа $N$ конечного $p$-индекса группы $B$ такая, что $|k N|=|h M|$. Группа $G_{M N}$ является свободньпм произведением конечных $p$-груп с циклическим объединением и, следовательно, аппроксимируется конечными $p$-группами в силу теоремы Хигмена [7]. Поскольку $g \rho_{M N} \neq 1$, существует гомоморфизм $\rho$ групшы $G_{M N}$ на конечную $p$-группу такой, что $g \rho_{M N} \rho \neq 1$.

Предположим теперь, что $r>1$. Пусть для определенности

$$
x_{1}, x_{3}, \ldots \in A \backslash H, \quad x_{2}, x_{4}, \ldots \in B \backslash K
$$

Поскольку подгрупшы $H$ и $K \pi$-отделимы в сомножителях $A$ и $B$ соответственно, то существуют нормальные подгруппы $A_{1}$ и $B_{1}$ групп $A$ и $B$ такие, что $A / A_{1}$ и $B / B_{1}-$ конечные нильпотентные $\pi$-групшы и

$$
x_{1}, x_{3}, \ldots \notin H A_{1}, \quad x_{2}, x_{4}, \ldots \notin K B_{1} .
$$

Пусть $l$ - наименьшее общее кратное чисел $\left|h A_{1}\right|$ и $\left|k B_{1}\right|$. Так как все простые делители числа $l$ принадлежат множеству $\pi$, то по предложению 1 существуют нормальные подгрупшы $A_{2}$ и $B_{2}$ групп $A$ и $B$ соответственно такие, что $A / A_{2}$ и $B / B_{2}$ - конечные нильпотентные $\pi$-групшы и $\left|h A_{2}\right|=l=\left|k B_{2}\right|$.

Пусть $M=A_{1} \cap A_{2}, N=B_{1} \cap B_{2}$. Тогда $|h M|=l=|k n|$. Теперь можно рассмотреть свободное произведение с объединением

$$
\bar{G}=(\bar{A} * \bar{B} ; \bar{h}=\bar{k}), \quad \text { где } \bar{A}=A / M, \bar{B}=B / N, \bar{h}=h M, \bar{k}=k N, \bar{G}=G_{M N} .
$$

Из (1) следует, что

$$
x_{1}, x_{3}, \ldots \notin H M, \quad x_{2}, x_{4}, \ldots \notin K N .
$$

Поэтому элемент $g \rho_{M N}$ имеет в группе $\bar{G}$ несократимую запись длины $r>1$ и, следовательно, отличен от 1 .

Покажем, что групша $\bar{G}$ апроксимируется конечными разрешимыми $\pi$-групшами. Заметим, что $\bar{A}$ и $\bar{B}$ - конечные нильпотентные $\pi$-групшы. Пусть $p_{1}, \ldots, p_{s}-$ множество всех простых делителей их порядков. Обозначим через $U_{i}$ и $V_{i}$ подгруппы групп $\bar{A}$ и $\bar{B}$ соответственно, порожденные теми силовскими подгруппами этих груп, порядки которых не делятся на $p_{i}, i=1, \ldots, s$. Поскольку порядки $\bar{h}$ и $\bar{k}$ по модулю подгрупп $U_{i}$ и $V_{i}$ совпадают, можно рассмотреть свободное произведение с объединением $\bar{G}_{i}=\bar{G}_{U_{i} V_{i}}$ и гомоморфизм $\rho_{i}=\rho_{u_{i} v_{i}}: \bar{G} \rightarrow \bar{G}_{i}$. По теореме Хигмена [7] группа $\bar{G}_{i}$ аппроксимируется конечными $p_{i}$-групшами, поэтому существует гомоморфизм $\sigma_{i}$ групш $\bar{G}_{i}$ на конечную $p_{i}$-группу, инъективный на сомножителях $\bar{A} / U_{i}$ и $\bar{B} / V_{i}$. Пусть

$$
F=\bigcap_{i=1}^{s} \operatorname{Ker} \rho_{i} \sigma_{i}
$$

Тогда

$$
\bar{A} \cap F=\bigcap_{i=1}^{s}\left(\bar{A} \cap \operatorname{Ker} \rho_{i} \sigma_{i}\right)=\bigcap_{i=1}^{s} U_{i}=1, \quad \bar{B} \cap F=\bigcap_{i=1}^{s}\left(\bar{B} \cap \operatorname{Ker} \rho_{i} \sigma_{i}\right)=\bigcap_{i=1}^{s} V_{i}=1 .
$$


По теореме Х. Нейман (см., например, $[8$, c. 122$])$ подгрупша $F$ групшы $\bar{G}$ является свободной. Легко заметить, что $\bar{G} / F$ - конечная нильпотентная $\pi$-группа. Так как свободная группа $F$ аппроксимируется конечньми разрешимыми $\pi$-групами, то по предложению 3 группа $\bar{G}$ также обладает этим свойством.

Поскольку элемент $g \rho_{M N}$ группы $\bar{G}$ отличен от 1 , существует гомоморфизм $\rho$ группы $\bar{G}$ на конечную разрешимую $\pi$-группу такой, что $g \rho_{M N} \rho \neq 1$.

3. Доказательства теорем. Теорема 2 непосредственно вытекает из предложений 2 и 4.

ДокаЗАТЕЛЬСТво тЕОРЕмЫ 1 . Пусть $A$ и $B$ - свободные групп, $h$ и $k$ - неединичные элементы групп $A$ и $B$ соответственно, $G=(A * B ; h=k)$. Пусть $m$ и $n-$ наибольшие положительные числа такие, что уравнения $x^{m}=h$ и $y^{n}=k$ разрешимы в группах $A$ и $B$ соответственно.

Рассмотрим сначала случай, когда одно из чисел $m$ или $n$ равно 1 . Пусть для определенности $m=1$. Обозначим через $b$ элемент группы $B$ такой, что $b^{n}=k$. Тогда группу $G$ можно разложить в свободное произведение вида

$$
G=(C * B ; t=b), \quad \text { где } C=\left(A, t ; h=t^{n}\right) .
$$

Баумслаг [9] доказал, что если элемент $f$ свободной группы $F$ порождает свой централизатор в этой группе, то група $L=\left(F, r ; f=r^{k}\right)$ аппроксимируется конечно порожденньми нильпотентными группами без кручения.

Поскольку $m=1$, элемент $h$ порождает свой централизатор в групше $A$. Поэтому в силу отмеченного выше результата Баумслага группа $C$ аппроксимируется конечно порожденными нильпотентными групшами без кручения. Свободная группа $B$ также обладает этим свойством.

Пусть $p$ - простое число. Хорошо известно, что конечно порожденная нильпотентная группа без кручения аппроксимируется конечными $p$-группами. Поэтому групшы $C$ и $B$ аппроксимируются конечными $p$-группами.

Заметим, что подгрупша $(t)$ группы $C$ совпадает со своим централизатором. Поэтому если $x \in C \backslash(t)$, то $[x, t] \neq 1$. Поскольку группа $C$ аппроксимируется конечными $p$-группами, существует гомоморфизм группы $C$ на конечную $p$-групп, ядро которого не содержит коммутатор $[x, t]$. Этот гомоморфизм отделяет $x$ от $(t)$. Следовательно, подгрупша $(t)$ группы $C$-отделима.

Очевидно, что подгруппа $(b)$ группы $B$ совпадает со своим централизатором. Как и выше, легко убедиться, что подгруппа ( $b) p$-отделима.

Таким образом, свободные множители $C$ и $B$ групшы $G$ аппроксимируются конечно порожденными нильпотентньми группами без кручения и объединяемые подгруппы $(t)$ и $(b) p$-отделимы в соответствующих сомножителях. По предложению 4 группа $G$ аппроксимируется конечными $p$-группами.

Рассмотрим теперь случай, когда числа $m$ и $n$ отличны от 1 . Если числа $m$ и $n$ являются степенями простого числа $p$, то по теореме 2 группа $G$ аппроксимируется конечными $p$-групами. Пусть теперь одно из этих чисел, скажем $m$, не является степенью простого числа $p$. Обозначим через $q$ простой делитель числа $m$, отличньй от $p$. Тогда существует элемент $a$ грушы $A$ такой, что $a^{q}=h$. Обозначим через $b$ элемент группы $B$, для которого $b^{n}=k$. При любом гомоморфизме группы $G$ на конечную $p$-группу коммутатор $[a, b]$ переходит в 1 . Поскольку этот коммутатор отличен от 1 , группа $G$ не аппроксимируется конечными $p$-группами. Теорема 1 доказана. 
ДоКАЗАТЕЛЬСТво ТЕОРЕМЫ 3. Сохраним обозначения из теоремы 1. Очевидно, что группу $G$ можно разложить в свободное произведение вида $G=\left(A_{1} * B_{1} ; h=k\right) * F$, где $A_{1}, B_{1}, F-$ свободные группы, причем $A_{1}$ и $B_{1}$ конечно порождены.

Предположим, что группа $G$ аппроксимируется нильпотентными группами. Тогда группа $D=\left(A_{1} * B_{1} ; h=k\right)$ аппроксимируется конечно порожденньми нильпотентными группами, которые, в свою очередь, финитно аппроксимируемы. Поэтому группа $D$ аптроксимируется конечными нильпотентньми группами.

Обозначим через $m$ и $n$ наибольшие положительные числа такие, что уравнения $x^{m}=h$ и $y^{n}=k$ разрешимы в групшах $A_{1}$ и $B_{1}$ соответственно.

Покажем, что группа $D$ аппроксимируется конечными $p$-группами для подходящего простого числа $p$. Допустим противное. Тогда по теореме 1 числа $m$ и $n$ отличны от 1 и не являются степенями одного и того же простого числа. Иньми словами, найдутся различные простые числа $p$ и $q$, делящие числа $m$ и $n$ соответственно. Пусть $a$ и $b-$ элементы групा $A_{1}$ и $B_{1}$ такие, что $a^{p}=h, b^{q}=k$. Тогда при любом гомоморфизме группы $D$ на конечную нильпотентную группу отличньй от 1 коммутатор $[a, b]$ переходит в 1 . Это противоречит доказанной вьше аппроксимируемости группы $D$ конечными нильпотентньпи группами.

Таким образом, группа $D$ аппроксимируется конечными $p$-группами для подходящего простого числа $p$. Поскольку $G=D * F$, где групша $F$ свободна, то групша $G$ также аппроксимируется конечными $p$-группами. Теорема 3 доказана.

Автор выражает благодарность профессору Д. И. Молдаванскому за ряд ценных замечаний и советов при написании этой статьи.

\section{СПИСОК ЦИТИРОВАННОЙ ЛИТЕРАТУРЫ}

[1] Baumslag G. On the residual finiteness of generalized free products of nilpotent groups // Trans. Amer. Math. Soc. 1963. V. 106. P. 193-209.

[2] Dyer J. Separating conjugates in amalgamated free products and HNN-extensions // J. Austral. Math. Soc. Ser. A. 1980. V. 29. № 1. P. 35-51.

[3] Brunner A., Burns R., Solitar D. The subgroup separability of free products of two free groups with cyclic amalgamation // Contemp. Math. 1984. V. 33. P. 90-115.

[4] Gildenhuys D. One-relator groups that are residually of prime power order // J. Austral. Math. Soc. Ser. A. 1975. V. 19. P. 385-409.

[5] Stebe P. Conjugacy separability of certain free products with amalgamation // Trans. Amer. Math. Soc. 1971. V. 156. P. 119-129.

[6] Gruenberg K. W. Residual properties of infinite soluble groups // Proc. London Math. Soc. 1957. V. 7. P. 29-62.

[7] Higman G. Amalgams of $p$-group // J. Algebra. 1964. V. 1. P. 301-305.

[8] Линдон Р., Шупп П. Комбинаторная теория групп. М.: Мир, 1980.

[9] Baumslag G. On the residual nilpotence of certain one-relator groups // Comm. Pure Appl. Math. 1968. V. 21. №5. P. 491-506. 